\title{
MET NP_000236.2:p.V504L
}

National Cancer Institute

\section{Source}

National Cancer Institute. MET NP 000236.2:p.V504L. NCI Thesaurus. Code C162301.

A change in the amino acid residue at position 504 in the hepatocyte growth factor receptor protein where valine has been replaced by leucine. 